\title{
Sôbre a Formula de Miller das Faces Tautozonas (*)
}

\author{
EDUARDO A. SALGADO
}

Escola Superior de Agricultura «Luiz de Queiroz»

(*) Recebido para publicação em 14/6/60. 


\section{1. - INTRODUÇÃO}

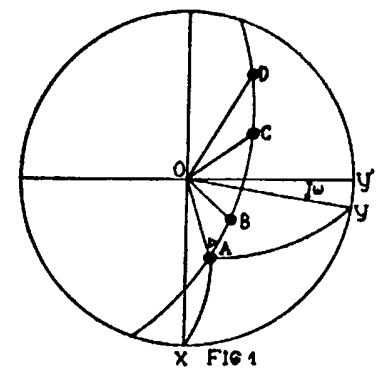

A muito conhecida e util fórmula de Miller tem sido deduzida de várias maneiras, por cristalografistas diversos. que segue.

Acreditamos seja nova a dedução

\section{2 - DEDUÇÃO}

$\mathrm{Na}$ figura 1 representa-se, em projeção estereográfica, uma zona contendo as faces A, B, C, D.

No plano do desenho estão situados os eixos cristalográficos $X$ e $Y$ e as coordenadas esféricas $\varphi$ são medidas a partir de OY', sendo $\omega$ $o$ ângulo formado por $O Y$ e $O Y^{\prime}$ e $\Delta o$ ângulo formado pelo círculo de zona e pelo círculo máximo passando pelos pontos $\mathrm{O}$ e $\mathrm{A}$.

$$
\begin{aligned}
\text { Tem-se, no triângulo } \mathrm{AOB}: \frac{\operatorname{sen} \mathrm{AB}}{\operatorname{sen}(\varphi \mathrm{A}-\varphi \mathrm{B})}=\frac{\operatorname{sen} \rho \mathrm{B}}{\operatorname{sen} \Delta} \ldots \\
\quad \text { e no triângulo } \mathrm{AOC}: \frac{\operatorname{sen} \mathrm{AC}}{\operatorname{sen}(\varphi \mathrm{A}-\varphi \mathrm{C})}=\frac{\operatorname{sen} \rho \mathrm{C}}{\operatorname{sen} \Delta} \ldots
\end{aligned}
$$

Dividindo, membro a membro, (1) por (2), vem:

$$
\frac{\operatorname{sen} A B}{\operatorname{sen} A C}=\frac{\operatorname{sen} \rho B(\operatorname{sen} \varphi \mathrm{A} \cdot \cos \varphi B-\operatorname{sen} \varphi \mathrm{B} \cdot \cos \varphi \mathrm{A})}{\operatorname{sen} \rho \mathrm{C}(\operatorname{sen} \varphi \mathrm{A} \cdot \cos \varphi \mathrm{C}-\operatorname{sen} \varphi \mathrm{C} \cdot \cos \varphi \mathrm{A})} \ldots
$$

Sendo $\left(h_{1} k_{1} l_{1}\right)$ o simbolo da face A e $m, n, p$ os cosenos diretores da face parametral, no sistema de eixos cristalográficos, o teorema dos cosenos de Wulff dá:

$$
\begin{aligned}
& \mathrm{h}_{1} \mathrm{~m}=\cos \mathrm{AX}=\operatorname{sen} \rho \mathrm{A} \cdot \operatorname{sen} \varphi \mathrm{A} \ldots(4) \\
& \mathrm{k}_{1} \mathrm{n}=\cos \mathrm{AY}=\operatorname{sen} \rho \mathrm{A} \cdot \cos (\varphi \mathrm{A}-\omega)=\operatorname{sen} \rho \mathrm{A}(\cos \varphi \mathrm{A} \cdot \cos \omega+ \\
& \operatorname{sen} \varphi \mathrm{A} \cdot \operatorname{sen} \omega) \ldots(5)
\end{aligned}
$$

De (4) tira-se:

$$
\operatorname{sen} \varphi \mathrm{A}=\frac{\mathrm{h}_{1} \mathrm{~m}}{\operatorname{sen} \rho \mathrm{A}} \ldots \ldots(6)
$$

Levando êste valor de $\operatorname{sen} \varphi \mathrm{A}$ em (5) obtém-se:

$$
\cos \omega A=\frac{k_{1} n-h_{1} m \cdot \operatorname{sen} \omega}{\operatorname{sen} \rho A \cdot \cos \omega}
$$


Para $\operatorname{sen} \varphi \mathrm{B}, \cos \varphi \mathrm{B}, \operatorname{sen} \varphi \mathrm{C}, \cos \varphi \mathrm{C}$ aparecem expressões idênticas à (6) e (7), e levando os valores assim obtidos em (3) vem:

$$
\frac{\operatorname{sen} A B}{\operatorname{sen} A C}=\frac{\left(h_{1} k_{2}-h_{2} k_{1}\right)}{\left(h_{1} k_{3}-h_{3} k_{1}\right)}
$$

Encontrar-se-á, anàlogamente:

$$
\frac{\text { sen } C D}{\operatorname{sen} B D}=\frac{\left(h_{8} k_{4}-h_{4} k_{3}\right)}{\left(h_{2} k_{4}-h_{4} k_{2}\right)}
$$

Tal tipo de dedução poderá ser repetido, òbviamente, para os indices $h$ e $l$ e $k$ e $l$, completando a fórmula.

\section{3. - RESUMO}

Apresenta o autor uma dedução nova, através da projeção estereográfica, da fórmula de Miller em que são interessadas faces em zona.

\section{4. - SUMMARY}

By means of the stereographic projection, the author presents a new deduction of Miller's formula concerning about the tautozonal faces.

\section{5. - BIBLIOGRAFIA}

BOEKE, H. E., 1911 - Die Anwendung der stereographischen Projektion bei kristallographischen Untersuchungen-Berlin - Verlag von Gebrüder. BARKER, T. V.-1922 - Graphical and tabular methods in cristallography - London - Thomas Murby \& Co. 
